\title{
From knowing to doing-from the academy to practice Comment on "The many meanings of evidence: implications for the translational science agenda in healthcare"
}

\section{Jo Rycroft-Malone*}

\section{Abstract}

In this commentary, the idea of closing the gap between knowing and doing through closing the gap between academics and practitioners is explored. The two communities approach to knowledge production and use, has predominated within healthcare, resulting in a separation between the worlds of research and practice, and, therefore, between its producers and users. Meaningful collaborations between the producers and users of research could in theory, create the conditions for more situated knowledge production and use, and result in a potential reduction in the evidence-practice divide within a health service context.

Keywords: Knowledge Translation, Co-Production, Implementation, Evidence-Based Healthcare

Copyright: (๑) 2014 by Kerman University of Medical Sciences

Citation: Rycroft-Malone J. From knowing to doing-from the academy to practice; Comment on "The many meanings of evidence: implications for the translational science agenda in healthcare". Int J Health Policy Manag 2014; 2: 45-46. doi: 10.15171/ijhpm.2014.08

\section{Article History:}

Received: 14 November 2013

Accepted: 2 December 2013

ePublished: 10 December 2013

Correspondence to:

Jo Rycroft-Malone

Email: j.rycroft-malone@bangor.ac.uk
$\mathrm{I}$ $\mathrm{n}$ order for us to better understand why there is a persistent challenge in ensuring safe and effective patient care, Harvey poses some questions for us to consider (1). These include questions about how the research-practice gap has been conceptualised, how different types of evidence are valued, and how knowledge is created. To date, the 'know-do' gap has been defined as a practice/service problem rather than a knowledge creation one, the conceptualisation of evidence has been relatively narrow, and there has been a lack of attention to the context or situation of evidence use. These are challenging issues, but ones we need to grapple with if we are to increase the chances of advancing 'our understanding of translational science for the benefit of patients, populations and health systems' (1).

Buying into the argument that evidence-informed practice is more than the use of research or its transformation in the form of guidelines or protocols would be hard for some to accept. These are epistemic issues; deep rooted and embedded in personal, group, professional, and organisational norms. Yet we know from observational research conducted in practice that practitioners rarely directly access formal knowledge (e.g. a piece of research or a guideline) in their everyday practice $(2,3)$. Professional work combines the tacit with the explicit (4). Therefore, if we wish to advance our understanding of translational science, perhaps we need to fix more of our attention on a better understanding of knowledge use in the reality of the practice context.

Harvey poses the question: 'What is the relationship between the producers and users of research?' Within the healthcare context, the two communities model of knowledge creation predominates in which the producers and users of research occupy separate worlds. Within the United Kingdom whilst there has been a huge investment in developing an infrastructure to fund and deliver high quality health related research, it has been argued that this has been achieved in part by splitting research production from the delivery of healthcare services (5). The impact of this has been an exacerbation of the boundary between research and practice and increasingly loud calls for a change of paradigm - from research production to knowledge mobilisation (5). Consequently, there has been a growing emphasis on practice-based perspectives to knowledge, which offers a contrast to an evidence-orientated understanding of knowledge use, which is predicated on an assumption that evidence is a product that needs to be pushed out to its users over the academic-practice boundary (6).

As there has been a growing recognition that improving health services and the pace of innovation requires a different sort of solution, the potential value of co-productive approaches has emerged. The notion of the creators and users of knowledge coming together to co-create solutions to real world problems is seductive; there would be no practice-academic boundary, evidence would be created within communities of practice, it would be of relevance to that community, and therefore, the gap between practice and research narrowed. In industry, such partnerships are common; in healthcare these have been less evident. However, there are now a number of initiatives in existence which have been created to provide the conditions and potential architecture for these types of collaborations within a health service context. For example, in the United States there has been the development of the Veterans' Health Administration Integrated Health and Research System and Clinical Translational Science Centres, and in England, a 
number of investments in Academic Health Science Centres and Networks, and in Collaborations for Leadership in Applied Health Research and Care (CLAHRCs).

The CLAHRCs offer an interesting natural experiment in bringing higher education institutions and health services closer together with the potential for this to provide a context for co-production and knowledge translation $(7,8)$. They are funded to conduct applied health research, implement findings from research into practice, and increase the capacity and capability of health services to engage with and apply research. The CLAHRCs have been subject to both internal and external scrutiny, and some interesting observations and lessons are beginning to emerge about their capacity to implement and mobilise knowledge. These include issues about structure and architecture, and power and positioning, which impact on CLAHRCs' ability to mobilise engagement and subsequently use knowledge. For example, whilst CLAHRCs were set up as joint academic-practice entities, in reality, different professional agendas foster the conditions for different levels of engagement. Different values, language and understanding between stakeholders, including a perception that CLAHRCs are dominated by the academic agenda, impacts on the shape and nature of the 'collaboration' (7) and thus their capacity for knowledge mobilisation.

CLAHRCs also demonstrate some interesting potential for collaborative relationships between the producers and users of evidence. This includes purposively setting up mechanisms such as investing in knowledge brokers, which can increase access to knowledge and enable a greater fit with the local context. Developing organisational facilitative structures and creating cognitive and physical 'spaces' can provide the opportunity and resources for different stakeholders to interact and problem solve, based on the different types of knowledge they bring to the discussion (5-7). Such observations demonstrate the potential of meaningful collaborations.

There has been a growing momentum for finding solutions to the challenging issue of providing consistently high quality, safe, and evidence-based patient care and service delivery. There is an emerging trend to find these solutions in a partnership or collaborative-based context, rather than in contexts that exaggerate the boundary and gap between research and practice. Such contexts have the potential to create the conditions in which multiple sources of evidence can be brought together and their relevance to local problems and contexts considered. Bringing academics and practitioners closer together is not without its own challenges. Differences in power and influence held by individuals and organisations, and across clinical and academic disciplines mediate the success of collaboration, and, therefore, the potential for knowledge mobilisation. However, in theory, a more joined-up approach should provide the opportunity for a situated approach to knowledge production and use, which takes account of context and stakeholder interests.

Ethical issues

Not applicable.

Competing interests

The author declares that she has no competing interests.

Author's contribution

JRM is the single author of the manuscript.

References

1. Harvey G. The many meanings of evidence: implications for the translational science agenda in healthcare. International Journal of Health Policy and Management 2013; 1: 1-2.

2. Gabbay J, Le May A. Practice-Based Evidence for Healthcare: Clinical mindlines. Abingdon: Routledge; 2011.

3. Dopson S, Fitzgerald L. Knowledge to action? Evidence-based health care in context. Oxford: Oxford University Press; 2005.

4. Schon DA. The Reflective Practitioner: How Professionals Think In Action. New York: Basic Books; 1983.

5. Walshe K, Davies HT. Health research, development and innovation in England from 1988 to 2013: from research production to knowledge mobilisation. J Health Serv Res Policy 2013; 18 : $1-12$.

6. Dopson S, Bennett C, Fitzgerald L, Ferlie E, Fischer M, Ledger $\mathrm{J}$, et al. Health care managers access and use of management research. Final report [internet]. 2012. Available from: http://www. nets.nihr.ac.uk/projects/hsdr/081808242

7. Rycroft-Malone J, Wilkinson J, Burton C, Harvey G, McCormack B, Graham I, et al. Collaborative action around implementation in Collaborations for Leadership in Applied Health \& Care (CLAHRCs): towards a programme theory. J Health Serv Res Policy 2013; 18: 13-26.

8. Currie $G$, Lockett $A$, Enany NE. From what we know to what we do: lessons learned from the translational CLAHRC initiative in England. J Health Serv Res Policy 2013; 18: 27-39. 